\title{
«Ce n'est pas vivre que d'exister dans un temps comme celui-ci » Gaston de Lévis : les lettres contre le néant
}

"It is not a life only to exist in a time like this one » Gaston de Levis: letters

against nothingness

\section{Laure Lévêque}

\section{(2) OpenEdition \\ Journals}

\section{Édition électronique}

URL : https://journals.openedition.org/ahrf/12859

DOI : 10.4000/ahrf.12859

ISSN : $1952-403 X$

\section{Éditeur :}

Armand Colin, Société des études robespierristes

\section{Édition imprimée}

Date de publication : 1 septembre 2013

Pagination : 81-99

ISBN : 9782200928261

ISSN : 0003-4436

\section{Référence électronique}

Laure Lévêque, « «Ce n'est pas vivre que d'exister dans un temps comme celui-ci » Gaston de Lévis : les lettres contre le néant », Annales historiques de la Révolution française [En ligne], 373 | juilletseptembre 2013, mis en ligne le 01 septembre 2016, consulté le 01 juillet 2021. URL : http:// journals.openedition.org/ahrf/12859; DOI : https://doi.org/10.4000/ahrf.12859 


\title{
"CE N'EST PAS VIVRE QUE D'EXISTER \\ DANS UN TEMPS COMME CELUI-CI » \\ GASTON DE LÉVIS : LES LETTRES CONTRE \\ LE NÉANT
}

Laure LÉVÊQUE

\begin{abstract}
À partir des lettres que le duc de Lévis adresse à sa jeune épouse sous la Révolution, cet article s'attache à mettre en évidence les convergences de cette écriture épistolaire avec un corpus romanesque plus tardif, qui court du René de Chateaubriand à $\mathrm{La}$ Comédie humaine, notamment pour ce qui est de la figure de l'aristocrate, puis de l'émigré, porteuse de contestation, refuge de l'opposition au réordonnancement social sorti de la Révolution. En confrontant l'écriture du for privé à l'appareil conceptuel élaboré, pour le roman, de Georg Lukács à Pierre Barbéris, se découvre, au-delà de la divergence générique, la continuité de l'écriture et des questionnements qui la traversent, qui sont ceux de la modernité bourgeoise et de ses contradictions, aux sources du romantisme.
\end{abstract}

Mots-clés : aristocrate, bourgeois, (contre-)révolution, émigré, romantisme

Dans l'Essai sur les révolutions, son premier ouvrage, fruit de trois années de travail que Chateaubriand publie à Londres, en émigration, en 1797, l'auteur, dont l'objet, sous la démarche comparatiste qui dit son ambition taxinomique, est de parvenir à s'expliquer cette révolution «qui n'eut jamais d'exemple » au point qu'elle en est à jamais singulière la Révolution - légifère en matière d'usage de la parole, renvoyant les témoignages à leur partialité : «On peut parler des choses passées ; mais quiconque n'est pas spectateur désintéressé des événements actuels doit se taire ». Avant d'ajouter : «Et où trouver un tel spectateur en Europe ? 
Tous les individus, depuis le paysan jusqu'au monarque, ont été enveloppés dans cette étonnante tragédie $\gg^{1}$. Une pétition de principe qui, si elle était observée, vouerait d'emblée les Lettres à Pauline de Gaston de Lévis (1764-1830) au néant. N'était que, en la transgressant lui-même, Chateaubriand sanctionne bien plutôt qu'avoir vécu la Révolution ne suffit pas à la comprendre quand comprendre la Révolution, c'est d'abord l'écrire, condition sine qua non pour s'approprier un objet si singulier, dans la distance que restitue l'écriture, à défaut du recul temporel.

Récemment éditées par les soins de Claudine Pailhès ${ }^{2}$ après qu'elles eurent fait l'objet d'une première publication sélective par un descendant de l'auteur, Antoine de Lévis Mirepoix (1884-1981), sous le titre «Une correspondance amoureuse et politique à la fin du $18^{\mathrm{e}}$ siècle $»^{3}$, ces lettres constituent pourtant un document d'importance considérable dans l'appréhension de la conjoncture révolutionnaire par un individu singulier, et ce d'autant plus que l'énonciation épistolaire - à l'inverse de l'écriture mémorialiste, qui emporte avec elle la remise en ordre a posteriori de la logique événementielle - restitue la trame des événements dans leur immédiateté, livrant accès aux états de conscience du scripteur. D'autant que le prix de ces lettres vient aussi de leur totale liberté de ton - qu'atteste la comparaison avec d'autres corpus similaires -, liberté de ton qu'il faut elle-même rapporter à la personnalité du jeune duc et à la relation faite de confiance, d'amour et d'abandon qui le lie à sa jeune épouse ${ }^{4}$ (1771-1829) - « il n'y a pas un repli de ma conscience où tu ne puisses pénétrer », lui

(1) François-René de CHATEAUBRIAND, Essai sur les révolutions, dans Essai sur les révolutions, Génie du christianisme, Défense du génie, Paris, Gallimard, «Bibliothèque de la Pléiade », 1978 , p. 43.

(2) Gaston de LÉvis, Écrire la Révolution : 1784-1795, « Lettres à Pauline », Cahors, La Louve Éditions, 2011. Toutes nos références, données entre parenthèses dans le corps du texte, renvoient à cette édition.

(3) La Revue de France, ${ }^{\circ} 14-15,1929$.

(4) Laquelle relation frappe par sa modernité. Dans la conception du couple qu'elle engage, qui ne suppose pas de subordination à la domination masculine (189, 328-329), Gaston se faisant un principe de traiter d'égal à égale avec ce « petit Robespierre femelle » (335) pétri d' « une idée d'égalité chimérique, de liberté hors de nature » à qui il écrit que leur mariage représente « l'époque dont je date pour la vie » (209) et qu'il la regarde comme sa «patrie » (365). Les termes, assurément, sont forts, qui rendent compte d'une relation dont la profondeur est peu commune et qui ne fera que se renforcer quand l'attachement conjugal se verra consolidé des liens de la paternité, avec la naissance d'une petite Augustine en décembre 1788, ce « toutou » qui n'est pas encore né que son père lui adresse des lettres (204-205), puis, en avril 1794, d'un héritier. Des entrailles paternelles du duc de Lévis, les preuves sont si nombreuses qu'il nous faut renoncer à signaler les pages où elles se manifestent. 
écrit-il le 8 août $1787(213)^{5}$-, destinataire de cette correspondance riche de 236 lettres $^{6}$ qui s'échelonnent de juin 1784 à décembre 1795.

Un individu singulier qui n'est pas, néanmoins, sans être socialement déterminé : duc, il tient à une grande famille de la noblesse languedocienne liée à la famille royale, lui-même étant colonel des carabiniers de Monsieur ${ }^{7}$ et son mariage, en 1784, avec une demoiselle d'Ennery, fille de planteurs martiniquais, l'a mis en puissance d'intérêts aux Antilles, toutes positions qui ne peuvent pas ne pas peser sur ce qu'il vit. Une alliance qu'il noue dans le milieu libéral, son beau-père, Victor Charpentier d'Ennery étant pleinement représentatif de cette classe d'hommes nouveaux dont les ancêtres ont commencé Charpentier - quoique bouchers - au XVII ${ }^{\mathrm{e}}$ siècle, avant d'intégrer la haute fonction publique, lustrés en d'Ennery par une savonnette à vilains qui fonde le dernier de leurs rejetons à devenir gouverneur de la Martinique où il se montre sensible à la condition des esclaves, certains de ses parents étant frottés d'égalité jusqu'à se faire les défenseurs du droit de vote pour les gens de couleur libres. Des positions que Gaston de Lévis est loin d'épouser tout uniment, qui se félicitera de ce que la Constituante ait rendu, le 24 septembre 1791, un décret qui abolisse celui du 15 mai portant l'égalité des Blancs et des Noirs $\left(372^{8}\right)$ : les affaires sont les affaires.

C'est qu'aux ducs ses pères font pendant les ducats de la lignée maternelle, toute entière vouée au négoce et à la marchandise. Une double ascendance de croisés et d'armateurs qui n'est sans doute pas sans prédisposer Gaston de Lévis aux voies de l'hybridation. Porteur du double héritage de la morale impérieuse des patriciens et de la souple philosophie des entrepreneurs, il préfigure ces types de métis sociaux qui fascinent Balzac, lequel en suit le destin, dans la France révolutionnée, à travers la thématique du mariage, qui cristallise les convergences objectives, les assimilations, les collusions. Or, dans le roman familial des Lévis, le tabou de dérogeance est de peu de poids au regard des enjeux financiers qui entourent l'union avec Pauline, fille unique et héritière d'une belle fortune, comme l'est aussi sa mère, qui a de solides espérances dans les îles tandis que, de son côté, seul fils, Gaston a hérité de sommes substantielles à la mort de son père, en 1787.

(5) Et encore le 10 avril 1792, « je ne craindrai jamais que tu y lises [dans mon cœur] » (385).

(6) Sur un ensemble qui en comportait 294. Cf. les précisions de Claudine PAILHÈs, dans Gaston de LÉvIS, Écrire la Révolution : 1784-1795, op. cit., p. 9.

(7) Depuis 1782, après avoir été fait, à 16 ans, capitaine des gardes du corps de Monsieur.

(8) «L'on a rendu hier un excellent décret sur les colonies, sage et juste ». 
La contrariété de ces traditions situe Gaston de Lévis dans la double mouvance du Prince et du Marchand, cette configuration symbolique que Pierre Barbéris ${ }^{9}$ reconnaît opératoire dans les Lettres et qu'il voit polariser, sous les espèces de ce couple de figures antagonistes, le mode d'être de chacune des classes dont la Révolution, périmant la société d'ordres, a consacré l'émergence : aristocrates et bourgeois ${ }^{10}$. Aristocrate le Prince, farouche défenseur d'une morale inflexible, à tout sacrifice prêt dès que l'honneur se trouve engagé, champion de la valeur, et bourgeois le Marchand ${ }^{11}$, qui ne croit qu'à l'utile et, comme tel, estime à l'aune du rapport jusqu' aux rapports humains en même temps qu'il remotive la valeur pour la réduire à la seule valeur d'échange.

Et c'est bien cette dualité, pour ne pas dire ce dualisme, qui subsumant ancienne et nouvelle France - désigne les deux voies offertes à la France de demain qu'enfante la Révolution et qui habite Gaston de Lévis.

Prince $^{12}$, Gaston de Lévis l'est absolument. Il l'est, non pas d'être né noble, mais d'agir noblement, conformément au principe qui fonde l'essence même de la noblesse tel que l'a formalisé Montesquieu : l'honneur. Montesquieu qui pose que, si ce fondement « se trouve choqué, il exige ou permet qu'on se retire chez soi. Il veut qu'on puisse indifféremment aspirer aux emplois, ou les refuser ; il tient cette liberté au-dessus de la fortune même ${ }^{13}$. Un «même » déjà lourdement indiciel des mutations engagées, comme autant de menaces pour les principautés héroïques.

(9) Pierre BARBÉRIS, Le Prince et le Marchand. Idéologiques, la littérature, l'histoire, Paris, Fayard, 1980.

(10) Voir du même Pierre BARBÉRIS, Aux sources du réalisme : aristocrates et bourgeois, Paris, UGE, 10/18, 1978.

(11) Sans que cette caractérisation épouse forcément la division sociale « réelle », l'écrit touchant à une réalité symbolique. Témoin Lucien Leuwen, que Stendhal choisit fils de banquier sous le régime du roi bourgeois et dont il fait néanmoins une haute figure de la résistance aux compromissions. Par là, il radicalise encore les positions d'un Julien Sorel, fils de charpentier et, comme tel, pouvant avoir objectivement intérêt à combattre l' «aristocratie chrysogène » (CHATEAUBRIAND, Mémoires d'outre-tombe, I, Paris, Gallimard, «Bibliothèque de la Pléiade », 1951, p. 277), comme il ne l'envoie pas dire à ses juges, lors de son procès. C'est que la rédaction du Rouge et le Noir, dont Stendhal corrige les épreuves lors des journées de 1830, précède la révolution alors que celle de Lucien Leuwen, entrepris en 1834-35, baigne dans l'affairisme louis-philippard et qu'il n'y a plus moyen de se faire illusion sur les ressorts qui meuvent la société : «Après la révolution de Juillet, lorsque le banquier libéral Laffitte conduisit en triomphe son compère le duc d'Orléans à l'Hôtel de ville, il laissa échapper ces mots : "Maintenant, le règne des banquiers va commencer ". Laffitte venait de trahir le secret de la révolution », Karl MARX, Les Luttes de classes en France, 1848-1850, dans Manifeste du parti communiste suivi de Les Luttes de classes en France, Paris, 10/18, 1962, p. 68.

(12) L'usage de la majuscule, allégorisant, situe au plan des représentations symboliques le contenu recouvert par Prince et Marchand, dans la logique des travaux conduits par Pierre Barbéris.

(13) Montesquieu, De l'Esprit des lois, Paris, Gallimard, « Folio », 1995, 1, p. 133-134. 
D'autant que si Montesquieu conclut au droit de retrait des aristocrates, dans son analyse, cette sécession entre en corrélation étroite avec la mise en cause de l'État monarchique et le renouvellement de sa base sociale, appuyé sur les capacités du Tiers, au nom de l'idéologie aristocratique $^{14}$. Au reste, c'est bien au moment d'envisager une redéfinition de la communauté nationale, dans ce qui touche à la recomposition du pays légal dont les États généraux, en s'arrêtant sur la représentation, questionnent la relation avec le pays réel, que, le 27 mai 1789, l'invitation du Tiers au clergé à se réunir à lui cristallise pour Lévis l'altérité aristocratique, vécue dans le malaise et la dissidence. Dès lors c'est dès l'origine, et n'importe la disjonction qu'introduit l'usage différencié des pronoms entre singulier et collectif, qu'à Lévis « La scission paraît inévitable, quelque parti qu[e le clergé] prenne. Nous touchons à de grands malheurs. Ma position va devenir bien délicate. Enfin, tant que l'honneur et toi me resteront, je ne serai pas malheureux » (250). Altérité aristocratique où le duc, dans l'étagement des sphères d'intervention du sujet - de l'ipséité (moi), à la vie privée (moi+toi) et à la société (moi $\subset$ nous) -, se reconnaît comme être de classe tout en se séparant comme conscience, douloureux divorce qui fonde le Prince quand cette double allégeance s'éprouve aporétique. C'est là, pourtant, ce qui gouverne Gaston de Lévis tout au long de la période, tant que les contradictions que feront surgir les événements s'avéreront gérables, entendre compatibles avec l'honneur.

Mais, pour être ne varietur, une telle ligne de conduite va vite tenir de la ligne brisée. C'est ainsi qu'il faut comprendre la position délicate dans laquelle la fidélité qu'il doit à Monsieur plonge notre épistolier. Et ceci, non pas seulement extérieurement, vis-à-vis du monde, par souci du qu'en dira-t-on dont bruissent les cercles étroits du faubourg Saint-Germain, mais bien intérieurement, à ses propres yeux, en son for intérieur. Ses lettres donnent clairement à lire les impératifs contradictoires avec lesquels il se trouve contraint de composer pour ménager ce à quoi il tient et faire souffrir le moins possible ses positions, pour rester fidèle, dans le présent de la conjoncture, à ce que prescrit l'honneur tout en conservant, à lui-même comme, plus encore, aux siens, un avenir, lequel ne peut demeurer ouvert qu'en se conformant à ce que Chateaubriand appellera plus tard la « morale des devoirs $»^{15}$. Parmi lesquels il faut compter ceux de sa charge. Envers

(14) Cf. Arlette JounnNa, Le Devoir de révolte. La noblesse française et la gestation de l'État moderne (1559-1661), Paris, Fayard, 1989.

(15) Dans un article fameux paru dans Le Conservateur du 3 décembre 1818, où il l'oppose à la «morale des intérêts », devenue hégémonique. 
Monsieur d'abord, dont il est le favori d'août 1788 à avril $1789^{16}$, et qu'il sert sans en être la dupe jusqu'à ce que la fuite à Varennes, dans le secret de laquelle il n'est pas mis, ne les sépare, ouvrant les voies à sa démission, le 4 juillet 1791 : « Vous savez bien que, s'il avait jugé à propos de m'emmener, je l'aurais suivi jusqu'à la frontière et que je serais revenu à mon poste ». Démission qu'il a le bonheur de pouvoir donner «motivée noblement » (341) en même temps qu'elle fait justice à ce qu'il percevait et condamnait le 14 mars de la dérive du comte de Provence vers le «cagotisme ${ }^{17}$ : «mon parti est pris : à la première mesure antipatriotique, je le quitte avec éclat et ne le verrai plus de ma vie » (315-316). C'est que la fidélité à sa race et sa classe n'exclut en rien le service de la patrie, dont il est un représentant, membre de la Constituante après avoir siégé aux États généraux. Sans plus d'illusion d'un côté que de l'autre, mais noblesse oblige, puisque c'est le souci de s'attirer la « considération » qui, toujours, règle sa conduite $(219,438)$. Noblesse oblige, cette impérieuse prescription que s'incorporeront les héros romantiques jusqu'à lui donner la force d'une devise, au risque de paralyser toute action, pire, d'un clivage du moi quand l'individu se voit écartelé entre des injonctions antinomiques. C'est là la symptomatique qui hante tout le premier romantisme, de René (1802) ou d'Oberman (1804) au romanesque de 1830 dont l'Octave de Malivert de Stendhal, «le devoir incarné $»^{18}$, offre, en 1827, l'expression la plus aboutie. Mais est-ce si différent hors la fiction ? Pour le duc de Lévis, qui n'a pas d'autre credo et répète à l'envi : « je sais très bien [...] ce que prescrit le devoir et surtout l'honneur qui est [...] le premier devoir » (360), la place qu' occupe Varennes dans son parcours idéologique, entre les deux loyalismes inconciliables qui le réclament, fait pencher pour la négative.

Après la fuite à Varennes, devenue, sous la plume désapprobatrice de Lévis, «l'équipée du roi », «les temps sont bien changés» (342). Jusque-là, Lévis peut marcher avec son temps et, nourri au Rousseau du Contrat social, se flatter de «devenir un petit Custine » (261), souscrire aux processus en cours et ne pas trouver sa praxis de représentant de la nation en désaccord avec son éthique aristocratique de l'honneur. C'est le temps de l'élan. Celui où, pressé d'exprimer «[s]es idées belles et neuves » (259), le 16 mai 1790, le duc « guette la parole » à l'Assemblée pour prendre part au débat où la Constituante discute de l'initiative de la

(16) Cf. p. 233, 236, 240, 242, 246, 252-253.

(17) Et, déjà, le 5 février 1791. Cf. aussi p. 296, 322, 324.

(18) STENDHAL, Armance, Paris, Gallimard, «Folio », 1975, p. 50. 
guerre $^{19}$ et proposer une motion : «L'A-[ssemblée].N-[ationale] déclare de la manière la plus solennelle que jamais la nation française n'entreprendra rien contre les droits et la liberté d'aucun peuple ; mais qu'elle repoussera avec tout le courage et l'énergie d'une grande nation libre et puissante les attaques de ses ennemis » (259), amendement qui, combiné aux vues de Volney, sera à l'origine de la Déclaration de paix au monde qui porte que « la Nation française [...] n'emploiera jamais ses forces contre la liberté d'aucun peuple ». Et ami du peuple, Lévis l'est aussi à l'intérieur, quand l'examen du découpage électoral le voit se ranger sans hésiter contre le décret du marc d'argent aux côtés de Pastoret et de Robespierre ${ }^{20}$ pour dénoncer le point de vue de Sieyès, et défendre à la tribune : « J'opine [...] pour que les bras soient représentés, non les écus $»^{21}$. Par où il se rencontre aussi avec Marat, féroce contre ce décret félon qui organise le reniement des conquêtes de l'égalité dont il affiche l' " oubli de tous les principes » pourtant posés par les législateurs eux-mêmes.

Mais la fuite du roi, avec ce qu'elle précipite, polarise les contradictions, que Lévis apercevait déjà entre la Constitution et la tranquillité publique (341), contradictions qu'il pointe entre les «principes constitutionnels d'inviolabilité » qu'il « regarde comme un des boulevards de la liberté nationale » (342) et leur application au monarque. Viciée, la Constitution l'est d'être assise sur des « institutions demi-philosophiques et demi-monarchiques » (353) et son dualisme a pour résultat de partager la nation en factions et de déchirer tout homme de bonne volonté en homo duplex. Par où elle acte aussi les embarras d'une politique du moyen terme. Les temps ne sont pas encore au juste milieu mais, bien plutôt, actualité de l'antique oblige, à cette «loi de Solon» (488) qu'évoque le duc, et dont Plutarque rappelle qu'elle « note d'infamie tout citoyen qui, dans une sédition, ne se déclare pour aucun parti. Apparemment il ne voulait pas que les particuliers fussent indifférents et insensibles aux calamités publiques, et que, contents d'avoir mis en sûreté leurs personnes et leurs biens, ils se fissent un mérite de n'avoir pris aucune part aux maux de la patrie. Ils

(19) Débat qui s'instaure à la faveur de frictions entre l'Espagne et l'Angleterre qui, si elles devaient déboucher sur la guerre, pourraient entraîner l'intervention de la France aux côtés de l'Espagne, son alliée.

(20) Voir son discours du 20 avril 1791 «Sur la nécessité de révoquer les décrets qui attachent l'exercice des droits du citoyen à la contribution du marc d'argent ou d'un nombre déterminé de journées d'ouvriers », Archives parlementaires de 1787 à 1860, Première série (1789-1799), tome XI, Paris, Librairie Administrative Paul Dupont, 1880, p. 320.

(21) Cité par Jacques DUPÂQUIER, Souvenirs-portraits du duc de Lévis suivi de Lettres intimes de Monsieur comte de Provence au duc de Lévis, Paris, Mercure de France, "Le Temps retrouvé », 1993, p. 18. 
voulaient que, dès le commencement de la sédition, ils s'attachassent à la cause la plus juste ; et qu'au lieu d'attendre de quel côté la victoire se déclarerait, ils secourussent les gens honnêtes $»^{22}$.

Or, quand bien même le roi s'est rendu coupable d'une faute politique, le seul tort de la famille royale " est d'avoir voulu jouir de cette liberté au nom de laquelle on les enchaîne » (342). On peut lui imputer « faiblesse » et « fausseté », d'avoir failli moralement donc, mais non point d'être sorti du cadre de la légalité, quand l'Assemblée, elle, viole maintenant la Loi en trahissant les principes garantis par la Constitution. Aussi, plus qu'une fidélité à une monarchie sur laquelle il ne se fait guère d'illusion, ce sont les vexations infligées à la personne du roi, en ce qu'elle éclairent les contradictions - grosses de déviation, pour ne pas dire de dévoiement qui traversent le processus révolutionnaire, qui jettent Lévis, de plus en plus sensible aux «injustices partielles », «désordres » et «atrocités » (343), dans la résistance en autorisant qu'il se désolidarise de la Révolution, s'il faut la confondre avec l'interprétation que lui donne désormais « le peuple », qui raccourcit des innocents qui en appellent à « la soumission aux lois » (349), ou « la canaille » à drapeau rouge qui se répand sur le Champ de Mars (349) et s'en prend à la garde nationale, avant qu'elle ne tire, provoquant le massacre du 17 juillet 1791 . Clairement, le duc se range maintenant parmi « les amis de l'ordre $»^{23}$ et sympathise « avec tout ce qu'il y [a] de gens honnêtes et bien vêtus » (349) contre ceux qu'il ne regarde plus que comme des « coquins » (451).

Mais la cause des gens honnêtes, chère à Solon, ne se laisse pas si aisément cerner. Elle est nationale le 23 juillet 1791, quand le duc mande à Pauline :

« Vous me connaissez assez pour savoir que je n'aime pas le despotisme, c'est pour lui, quoi qu'ils disent, que les princes viendront combattre, vous voyez donc bien que je serais entre le marteau et l'enclume, craignant également le succès des fous pour lesquels je me battrais et des tyrans que j'attaquerais. Ainsi, je me regarderai comme le plus heureux du monde si je puis être spectateur tranquille de cette querelle, mais j'ai bien peur que l'on n'attaque avant la fin de l'Assemblée, alors, la neutralité devient impossible

(22) Plutarque, Vies des hommes illustres, Vie de Solon, XXV, trad. D. RICARD.

(23) Un ralliement auquel Lévis ne se résout pas sans tiraillement. Après le massacre du champ de Mars, il ne fonde plus guère d'espoir sur la responsabilité du parti royaliste et son pronostic va à une Charte constitutionnelle approuvée par le roi avec force discours lénifiants, avant une nouvelle fuite, qui ne laisse d'autre issue que la guerre : « et tout fondra sur la malheureuse France » (354). Des prévisions où la référence aux « amis de l'ordre » est cette fois clairement dépréciative. 
puisqu'il faudrait fuir et c'est ce que je ne ferai jamais, je servirai donc dans l'armée patriotique » (353-354).

«Il y a plus de choses dans le ciel et sur la terre que n'en peut rêver votre philosophie », comme le disait Hamlet, Prince par excellence et, singulièrement, des royaumes où il est quelque chose de pourri, prince dépossédé à qui ne restent que les ressources, critiques, de l'intellectuel, et dont le romantisme fera sa figure tutélaire ${ }^{24}$. En particulier, est ici déclassée la philosophie épicurienne, chère à une fraction de la noblesse éclairée et libre penseuse. Adieu Suave mari magno, dont passe ici l'écho et le regret ${ }^{25}$. Aussi en août 1792, convaincu par des bruits fallacieux de ce que l'ennemi a franchi la frontière, un Lévis très marqué par la journée du 20 juin peut-il écrire, au terme d'un revirement dans lequel l'affaire de Varennes aura été cruciale en ce qu'elle justifie le tournant idéologique contre-révolutionnaire qu'il prend et, avec lui, tous ceux parmi les nobles qui n'étaient pas hostiles aux réformes mais demeurent prisonniers de positions de classe : « il faut espérer que cette maudite révolution sera étouffée bien vite » (453). Au milieu des intrigues qui annoncent le duc de Brunswick à Paris à l'été 1792, Lévis lève le masque, mais pour sa femme, en même temps qu'il s'efforce de le prendre pour la société : «j'ai dû dire (ce qui est vrai) [...] que je ne désirais pas la contre-révolution ». Et d'aussitôt modaliser : « ou plutôt que je ne voulais pas en tirer d'autre parti que celui de manger mon bien dehors, au moins 3 ou 4 ans » (415).

Or, prendre parti, on a vu que les circonstances l'exigeaient, mais tirer parti ? L'expression est d'un Marchand. C'est que, si, chez Stendhal, les rôles admettent une claire répartition que rend sensible la stricte division fonctionnelle des personnages - Octave est souverainement Prince et son oncle, le commandeur de Soubirane, tout chevalier de l'ordre de Malte qu'il est, un Marchand accompli ${ }^{26}$-, le réel ignore ces solutions de continuité : dans l'épaisseur tissue de contradictions de cette « opposition qui s'appelle

(24) $C f$. notamment Pierre BARBÉRIS, «Le prince, l'intellectuel, les pouvoirs », dans Le Prince et le Marchand, op. cit., p. 217-241.

(25) Lesquels retentissent en des termes étonnamment similaires jusque chez cet autre lecteur des philosophes, sectateur des idées nouvelles qu'est Lucien Leuwen, Prince par l'esprit, et ce d'autant plus qu'il lui faut racheter d'être Marchand par la naissance, lequel éprouve aussi cette singularité qui l'aliène à lui-même et lui interdit le monde : « Mon sort est-il donc de passer ma vie entre des légitimistes tous égoïstes et polis, adorant le passé et des républicains fous généreux et ennuyeux, adorant l'avenir ? Maintenant, je comprends mon père, quand il s'écrie : " Que ne suis-je né en 1710, avec cinquante mille livres de rente !", STENDHAL, Lucien Leuwen, 1, Paris, GF, 1982, p. 222.

(26) Tant il peut y avoir divorce entre la naissance objective et la conduite de fait des individus. De cela, la figure du commandeur de Soubirane telle que la constitue Stendhal dans son premier roman, Armance (1827), où il apparaît doublé d'un infâme spéculateur, est pleinement indicielle. 
la vie $\gg^{27}$, les choses ne sauraient être aussi tranchées et, si Lévis est Octave, il est aussi Soubirane, Marchand aussi bien que Prince.

Sa condition de Prince, Lévis y fait honneur en demeurant fidèle à ce qu'impliquent ses engagements de représentant de la nation, jusqu'à l'accomplissement de sa mission, le 30 septembre 1791 où l'Assemblée se sépare après avoir procuré à la France sa première constitution, et cela quelque risque qu'il puisse encourir ${ }^{28}$, sans compromission, sans se faire le «complice » (342) de ce à quoi il n'adhère pas, bravant la double adversité des Indigents, menés par Robespierre, dont Lévis voit bien qu'ils professent de « redoutables principes » qui « annoncent ouvertement le républicanisme » (338) et de ces contre-révolutionnaires « déraisonnables et désagréables » (494) « mécontents enragés qui soupirent après la combustion de leur pays » (324), dont il se démarque sans ambiguïté ${ }^{29}$. Signe de cette interprétation formaliste des devoirs, c'est sitôt la Constitution promulguée, début octobre 1791, que Gaston de Lévis passe la frontière rejoindre Pauline en Angleterre, où il demeure jusqu'à la fin mars 1792. Le vote de la loi sur les suspects, en novembre 1791, l'y trouve donc, pleinement lucide sur les vicissitudes de la fortune, qui déclare : «l'on sera obligé [...] de définir ce qu'est un émigré et je crains bien d'être un émigré » (376), et qui fait donc en toute conscience le choix d'en être un, en ne rentrant pas en France avant le terme prévu du 9 février 1792. C'est précisément en 1791 qu'apparaît, pour désigner les particuliers que les menaces révolutionnaires poussent à s'expatrier, le substantif «émigré », dérivé du verbe, dont l'usage l'emporte sur celui d' « émigration $»^{30}$, signe d'un désarroi qui est d'abord celui d'individus emportés dans des processus encore opaques. Et il est certain que cette condition, qui le frappe alors qu'il n'exerce plus aucune charge nationale, joue sur les frontières de la pensée pour rendre étranger Lévis à sa patrie, levant les freins qui arrêtaient sa conduite en solidarisant en lui la France et le Français. Plus question, dès lors, de servir dans l'armée patriotique,

(27) Balzac à sa sœur Laure, le 30 avril 1849, Correspondance, V, Paris, Garnier, 1969, p. 556.

(28) Ces risques, il les mesure, lui qui a éloigné sa femme de Paris dès la fin de l'année 1790 où Pauline gagne les Pays-Bas avant, sur ses instances, de rejoindre l'Angleterre au début de l'été 1791, seul moment où Gaston ressaisit l'autorité maritale pour interdire à sa femme tout retour en France dans des circonstances qu'il juge périlleuses.

(29) Ceux que, en mai 1792, même après la déclaration de guerre, en rien rallié à l'« imperturbable aristocratie » (415) de sa sœur, dans un clair effet de distanciation, il appelle « vos aristocrates » (294).

(30) Claire JAQUIER, Florence LOTTERIE, Catriona SETH (dir.), Destins romanesques de l'émigration, Paris, Desjonquères, «L'Esprit des Lettres », 2007, p 13. 
comme il l'avait résolu en juillet 1791, en avril 1792, c'est l'esquive qui prévaut : «il ne faut pas que je sois en France pendant la guerre de peur d'être obligé de la faire, il faut que je n'y sois pas au moment de la contre-révolution, il faut que je n'y sois pas après. Ainsi, il faut que d'ici là, j'y sois le moins possible, cependant toujours en conservant notre bien » $(387-388)^{31}$.

Qu'il rejoigne, le 14 août 1792, l'armée des Princes n'y pourra rien changer : Prince il ne l'est plus guère, celui que gouverne le seul souci de conserver son bien.

La convergence avec les figures littéraires que développera le romantisme frappe dans cet écartèlement d'un être héroïque acquis à la vertu aristocratique de l'honneur, valeur supérieure à la vie même, mais affronté à une indispensable adaptation dans une transition historique qui s'accompagne d'une transaction sur les principes quand le duc s'adonne bourgeoisement au mercantilisme. Dans ce moment historique si singulier, le duc et les siens illustrent la force pulsionnelle de la vie, entre crispation sur un monde qui s'abîme et aspiration à vivre quand même.

Mais convertir ce vouloir-vivre en pouvoir-vivre suppose de comprendre la Révolution et le cours qu'elle va prendre, aussi le duc exhorte-t-il continûment sa femme à l'économie : « personne ne sait ce que nous aurons et si nous aurons dans six mois. Je sais que je t'aimerai toujours, mais cela ne nourrit pas » lui écrit-il galamment fin 1790 (274), reconnaissant que « L'article qui est essentiel et celui qui m'inquiète le plus », «c'est ce malheureux argent » $(363)^{32}$. C'est que, « pour être heureux [...] il faut être dans l'aisance », ce qu'il estime, une fois le couple réuni, à 15000 livres de rente. Or, Gaston fait ses comptes : si, ces 15000 livres, ils les ont, il faut compter avec « des pillages, des séquestres, des confiscations, et je les crains également des deux côtés » (363). Aussi le duc, résolu à exploiter la valeur d'échange, met-il toute son industrie dans l'établissement, début 1791, d'une filature de $\operatorname{coton}^{33}$ qui connaît d'heureux débuts : « Nous filons tant que nous pouvons et nous changeons déjà du coton de 50 sous en coton

(31) Aussi s'il rentre en France, ce n'est plus pour prendre sa part des affaires de la patrie et travailler pour l'avenir, c'est « pour n'être pas ruiné » (2 avril 1792, 377). Une ruine qui admet toujours, chez Lévis, un sens spécifiquement financier. Ainsi, lorsqu'il annonce à Pauline : «Cette nouvelle de Chambéry [pris] et notre incroyable retraite me font de nouveau regarder notre ruine comme certaine et me dégoûte un peu plus (s'il est possible) de la vie », ce n'est pas de défaite qu'il s'agit mais de faillite : «Je ne pourrais pas vous voir privée de l'aisance et de l'agrément que vous méritez » (496).

(32) Lévis met l'accent sur « les finances (qui, dans le système moderne, sont tout) » (312). Il faut donc faire de la prospective, anticiper profits et pertes pour se ménager un fonds de réserve pour l'avenir.

(33) Voir les explications de Claudine PAILHÈs, op. cit., n. 106, p. 317. 
de 110 sous, mais cela ne va pas assez vite encore et nous voulons aller à 8 livres » (317). L'esprit d'entreprise atteint au miracle de la transsubstantiation qui, sous les espèces les moins spirituelles qui soient, convertit le coton en or et le Prince en Marchand. Alors, entrant sans complexe dans des « spéculations » (379) où il cherche à s'assurer la plus-value maximale, Gaston fait passer à Pauline, dès mars 1792, des tissus qu'elle est chargée d'écouler au prix qu'il a fixé : 15 guinées les voiles, 4 les corsages (382). S'appuyant sur une analyse du marché qui intègre une ethno-théorie du goût, Gaston projette de diversifier sa production (taffetas, linon, gaze...) et de spéculer sur les tentures «fadasses » qui plaisent tant aux Anglais : « Je crois qu'il y a prodigieusement à gagner sur cet article» (382). Si le textile demeure l'activité principale de l'entreprise, celle-ci s'étoffe, qui s'étend aux bijoux (391) et jusqu'au commerce du vin - du champagne -, pour lequel Pauline est commise (381), dirigée par un Gaston qui n'oublie pas de préciser que la marchandise doit reposer avant toute transaction (392). L'exploitation, il est vrai, a gagné en envergure -3000 bouteilles en juin 1792 - et exige un suivi attentif, aussi les lettres prennent-elles une nette coloration affairiste quand Lévis, au moment de Valmy, attend de sa femme qu'elle fasse état, dans chacune de ses lettres, du « taux du change de Londres sur Paris » (488).

Voici accomplie la métamorphose du duc en agioteur, laquelle n'engage en rien une mutation de degré, mais bien de nature, qui signe, une fois entamée, l'irrémédiable dégradation de l'être. Aussi le retrouvet-on songeant à des entreprises infiniment plus discutables. Ainsi après que l'Assemblée a, le 9 mars 1791, décidé d'examiner à Orléans le cas des criminels de lèse-nation, Lévis, prenant acte de ce que «Orléans se peuplera », aura cette peu chrétienne pensée : «j’ai envie (par spéculation) d'y louer des maisons » (24 mai 1792, 416).

Une spéculation qui étouffe tout respect humain quand le désintéressement le cède au calcul, périmant toute l'idéologie du sacrifice héroïque au fondement de la morale aristocratique. Dès lors, à Quiberon même, scène s'il en est du loyalisme sacrificiel, l'équipée, dont, sur les instances de sa femme, il est, n'inspire plus à Lévis qu'un désabusement distancié - «Certainement, je me dois à l'honneur avant tout, mais il me semble qu'il ne commandait point dans cette circonstance, vu la quantité de gens qui sont restés en Angleterre et ailleurs, et si cette aventureuse expédition qui ne monte pas à 3000 hommes effectifs est détruite, j'aurai en périssant le regret de n'avoir pas conservé un bon père à mes enfants dans un temps où il était encore plus nécessaire » (516), qu'allait-il faire dans cette galère ? - et une préoccupation comptable : touchera-t-il son dû ? «Il serait un peu 
injuste que je fisse la guerre à mes dépens tandis que tout le monde est grassement payé » (535). Une fois encore, l'homologie des questionnements interpelle - jusqu'à l'identité terminologique -, de l'écriture du for privé aux espaces de la fiction, quand le vécu de Lévis rencontre celui du comte de Fontaine, serviteur zélé du comte de Provence pour lequel il a payé de sa personne et de sa cassette pour se voir, une fois ce dernier revenu aux Tuileries, payé d'indifférence : « aucune de ces augustes personnes n'eut l'idée de lui demander le compte de ses pertes, ni celui de l'argent généreusement versé dans les caisses de l'armée catholique. Il s'aperçut un peu tard qu'il avait fait la guerre à ses dépens $»^{34}$.

Comme le comte balzacien, Gaston est bon lecteur de la Révolution. Et ce d'autant plus qu'il doit se faire l'instituteur de ses femmes. De sa tête folle de belle-mère surtout, qui a du mal à prendre la mesure de la situation. Si le séjour que ces dames d'Ennery ont fait en Brabant les a persuadées que la Révolution était étouffée, Gaston les détrompe, le 4 janvier 1792, en émigration :

«Vous prenez des idées très fausses de l'état de la France. Il y a une chose qui consolide tous les jours la révolution d'une manière singulièrement forte, c'est la vente des biens ecclésiastiques ${ }^{35}$. Elle se fait à un prix énorme, deux ou trois fois l'estimation. Dans plusieurs provinces, ce sont donc des capitalistes qui achètent de grosses masses et qui revendent en détail aux petits bourgeois et aux paysans. Chaque arpent de vendu fait un prosélyte et tel homme que la liberté ni l'intrigue n'aurait pu remuer se battra comme un Roland pour conserver son arpent » (279).

Roland petit bourgeois, Roland paysan! L'incongruité référentielle dit la violence de la dépossession. Celle-là même que Balzac suit dans ses Paysans dans la mutation du domaine des Aigues, passé de la mouvance de la famille royale au général de Montcornet, promu par l'Empire mais « fils d'un marchand du faubourg Saint-Antoine », avant le dépeçage en règle qui rend la terre aux paysans :

«La Révolution de 1789 a été la revanche des vaincus. Les paysans ont mis le pied dans la possession du sol que la loi féodale leur interdisait

(34) Honoré de BAlzaC, Le Bal de Sceaux, Paris, Furne, I, 1842, p. 2.

(35) Lorsque viendra le tour des émigrés, l'implicite de la mesure n'échappera pas au duc : « Il est clair que l'on veut englober le plus de monde possible dans la proscription pour avoir le plus de terre possible en supplément aux biens du clergé ». 
depuis douze cents ans. De là leur amour pour la terre qu'ils partagent entre eux jusqu'à couper un sillon en deux parts $»^{36}$.

Un amour qui, d'ailleurs, pour Balzac comme pour Lévis, qui dit compter avec « les attentats de cette législature ou d'une autre sur les propriétés territoriales (280) », ne va pas tant à la terre qu'à la propriété, donnée comme le véritable mobile de la Révolution et de son action. Les nouveaux Roland se sont trouvé une cause à leur mesure.

Car cette saisie contradictoire ne tient pas à la personne du duc qui, frappé d'aboulie, souffrirait de schizophrénie. Elle n'est pas davantage chue d'un désastre obscur : si désastre il y a, il n'a rien d'obscur, c'est celui même de l'HISTOIRE, du divorce entre HISTOIRE, Histoire et histoire ${ }^{37}$. Or le sens de l'HISTOIRE consacre le Marchand. Quid du Prince, alors, lâché dans un monde qui appartient désormais aux bourgeois conquérants ? L'histoire a tranché, dans la littérature, jusque tard dans le XIX ${ }^{\mathrm{e}}$ siècle $^{38}$, le Prince fait sécession: les tripotages boursiers ? La politique, dans un monde que gouvernent truqueurs et imposteurs (ô, Hamlet !) ? Les voies trompeuses de la vie ? Le mariage et la reproduction sociale ? Non possumus! Le Prince meurt, mais ne se rend pas. Mais l'Histoire fait toucher une tout autre réalité que ce réordonnancement fantasmatique qui orchestre la revanche symbolique de ceux qui ne se reconnaissent pas dans l'ordre des vainqueurs, fait entendre une tout autre lecture de l'HISTOIRE. Octave de Malivert appartient à l'histoire, Gaston de Lévis à l'HISTOIRE, mais ses lettres relèvent de l'Histoire. Mais quand les principautés littéraires peuvent se donner les gants de nier l'HISTOIRE en souscrivant à la binarité du dilemme : « Être vrai et courir à sa perte comme le Prince, ou vivre et durer dans le faux mais dans l'efficace (?) comme le marchand ? ${ }^{39}$, c'est là un luxe que, dans l'objectivité du réel, ne peuvent s'offrir leurs homologues, contraints de composer et de se colleter avec elle. De là le mariage - contre nature mais de raison - du Prince et du Marchand dont Lévis offre un exemple, avant les aristocrates de la Comédie humaine, moins rétifs à la sanction du réel que ceux de Stendhal.

(36) Honoré de BAlZaC, Les Paysans, op. cit., XVIII, p. 84.

(37) Je reprends là encore la distinction opératoire de Pierre BARBÉRIS entre HISTOIRE, qui renvoie au tissu événementiel, à « la réalité (?) historique », Histoire, qui en suppose la mise en ordre, et histoire qui, dans la fiction, « désigne ce que met en œuvre l'écriture, dans la saisie à la fois fantasmatique et raisonnée [...] de réalités qui n'ont pas encore droit [...] à l'Histoire, mais qui appartiennent quand même, qu'on le veuille ou non, on le verra un jour, à l'HISTOIRE », Prélude à l'utopie, Paris, PUF, 1991, p. 10.

(38) On pense au des Esseintes de Huysmans dans $\grave{A}$ rebours (1884).

(39) Pierre BARBÉRIS, Le Prince et le Marchand, op. cit., p. 11. 
Pourtant, l'hybridation qu'ils manifestent ne débouche sur nulle dialectique et aucun discours n'est à même de subsumer les contradictions pour ouvrir sur une position tenable. Lévis excluait de fuir et prévoyait de servir dans l'armée patriotique, il émigre et rejoint l'armée des Princes ; de sa vie il ne devait revoir Monsieur, coupable de trahison à Varennes, mais Pauline doit peu glorieusement solliciter sa protection... Ces revirements spectaculaires qui émaillent son parcours, l'adaptation à une conjoncture extrêmement mouvante ne les explique pas entièrement : dans cette dichotomie, c'est le romantisme qu'on reconnaît - dans ses empêchements, dans la dynamique de son vouloir-vivre que vient contrarier le pouvoir-vivre -, quand bien même il ne s'identifie pas pleinement au romantisme des vaincus dont Chateaubriand lancera le type avec René.

Ces romantismes, une ligne les partage, celle du réel : dans la littérature, qui œuvre à la compensation, on peut se faire Prince et porter l'idéal abstrait de la résistance aux compromissions ; dans le concret du réel, si l'on veut vivre, il faut que le Prince s'accommode du Marchand. Et c'est mortifère. Mais c'est la dynamique de la vie, de cette vie. C'est très exactement ce qu'éprouve un Lévis de plus en plus détaché de tout espoir d'agir sur le réel et, partant, de lui-même. Douché par la résistance du réel aux constructions idéelles, l'enthousiasme généreux de ce rénovateur attentif qu' aura authentiquement été Lévis ${ }^{40}$ au moins jusqu'à septembre 1791 se dessèche en un point d'honneur formaliste qui touche au conformisme. Oubliées les idées belles et neuves et la déclaration de paix au monde, il s'agit de se montrer sur le champ de bataille. Ainsi, « le grand point sera que l'on aura fait comme tous les autres, que personne n'aura plus rien à vous reprocher (30 août 1792, 467). C'est acquis le 25 septembre 1792, après Valmy : «alors, il me paraît que j'aurai satisfait à tout » (487). Et qu'importe qu'on n'y croie guère et qu'on n'agisse que mu par des motifs personnels : préserver son nom de toute tache et, par là, ménager un avenir aux siens.

L'avenir qui vectorise toute l'action du duc. Et non pas seulement celui de sa race, conformément à la tradition aristocratique, mais bien aussi son destin propre. L'histoire littéraire s'est longtemps plue à avancer que la Révolution n'avait pas soufflé sur les Lettres. C'est au moins à nuancer : la Révolution a retenti dans les Lettres comme la déclaration des droits

(40) Témoin, le 20 mai 1789, cette sortie contre un d'Epremesnil qui recherche le blocage, si impérieuse qu'il en interrompt un billet à Pauline : « je finis pour lui répondre vigoureusement car si nous allons épiloguer sur les mots, ce sera terrible et nous détruirons la conciliation qui sans cela commencerait peut-être à s'établir » (249). 
dans la société, en promouvant l'individu, en libérant le moi. Aussi, « Dans toute cette incertitude d'événements, il faut un peu penser à soi » (494). Et on y pense, en se félicitant que, même compromis pour avoir « été du côté gauche » (463), il se trouve des gens qui « s'obstinent à croire que je ne suis point fini » (415). Mais comme René aspirant aux espaces d'une autre vie, pressé de s'employer mais allant s'ensevelir au désert, c'est sous le mode de la disjonction que Lévis envisage le futur, balançant continûment entre sortir, qui est le fait du Prince, et entrer dans la carrière en sacrifiant à la course aux places comme un Marchand. Si tantôt il assure à Pauline vouloir se renfermer dans la vie privée, à l'abri du bruit d'un monde dont on ne partage pas la folie - « rassurez-vous, une fois ces grandes questions passées, je rentre dans le néant et revoler dans tes bras » (261), « se rejeter dans le tourbillon des affaires, c'est ce que je ne ferai pas. Je crois que sous un gouvernement modéré quelconque j'en aurais la possibilité, mais je doute fort que la volonté m'en revienne » (365) -, concurremment et inséparablement, il ne reste pas sourd à la voix de l'ambition : « je me jetterai dans la diplomatie » (487).

C'est d'ailleurs dans cette intrication entre une vie publique qui se fait, à mesure que la Révolution suit son cours, plus envahissante, et une vie privée qui a aussi ses exigences qu'il faut situer la spécificité de cette correspondance : c'est dans cette tension qui l'habite que réside sa dynamique. À ce titre, outre un document littéraire exceptionnel, ces lettres éclairent les débats intérieurs qui ont agité les consciences les plus lucides, dont le duc fait assurément partie, accompagnant la difficile gestion de prescriptions antagonistes en mettant en lumière l'émergence de modalités nouvelles d'inscription du sujet dans la Cité - lesquelles retentissent sur les mœurs ${ }^{41}$ et bouleversent les catégories de pensée -, l'urgence et la difficulté de s'y adapter. Il est dès lors moins surprenant de voir l'Histoire rencontrer l'histoire et le duc de Lévis se retrouver avec des nobles plus fictifs, tel le marquis de Saint-Alban de Sénac de Meilhan, qui cristallise les empêchements qui étranglent aussi un Gaston de Lévis embarqué dans une aventure qui dépasse sa personne autour de modalités de résistance qui informeront la sensibilité et l'imaginaire romantiques, dont l'émigré est le premier à fixer le type.

Que la première littérature romantique soit inséparable d'un rapport douloureux à l'ordre sorti de la Révolution, Chateaubriand ne dit pas autre

(41) À commencer par cette vie privée dont Balzac saisira qu'elle ne fait qu'intérioriser des mécanismes d'aliénation venus de la sphère publique. 
chose. En même temps qu'il montre que les succès des canonniers de Napoléon, soutenus par les canons du classicisme promus appareils de propagande du régime, ont considérablement retardé la constitution d'une esthétique romantique, confinée dans les milieux de l'émigration :

« La littérature qui exprime l'ère nouvelle, n'a régné que quarante ou cinquante ans après le temps dont elle était l'idiome. Pendant ce demisiècle elle n'était employée que par l'opposition. C'est madame de Staël, c'est Benjamin Constant, c'est Lemercier, c'est Bonald, c'est moi enfin, qui les premiers avons parlé cette langue. Le changement de littérature dont le dix-neuvième siècle se vante, lui est arrivé de l'émigration et de l'exil $»^{42}$.

Or Gaston de Lévis ressemble de bien près à ces héros des romans d'émigration qui connaissent aussitôt une vogue considérable. La frontière générique s'amenuise, que permet d'annuler la forme épistolaire élue par bien des romanciers, à commencer par Sénac, qui livre avec L'Émigré le parangon du type. Sénac qui s'attèle à la rédaction en 1793, au plus fort des polarisations, mais n'accède au geste de publication que sous le Directoire, en 1797, soit au moment où un discours de réconciliation est devenu tenable, comme en témoigne la démarche d'un Lally-Tollendal, auteur cette même année d'une Défense des émigrés français où il propose de reconnaître la république en échange d'une amnistie. Si la publication a été retardée jusque-là, les difficultés matérielles n'y sont pas étrangères, pour autant, il faut faire toute leur place à d'autres types d'empêchements, internes ceux-là, où le blocage procède non seulement de l'impossibilité de penser les événements à chaud, mais tient à la disponibilité même d'une idéologie de recours. Et il fallait bien attendre les années 1796-97 pour que l'Avertissement liminaire pût identifier un objet fédérateur en un certain " général, maître de l'Italie » auquel le narrateur se plaît à faire justice, moins peut-être pour la raison expressément alléguée - sa modération, qui contraste heureusement avec l'époque de la Terreur - que pour ce qu'il incarne, lui qui, jusqu' au bout, se dira «national », un socle commun de valeurs pour lesquelles tout Français pourra vivre et mourir.

En attendant, comme les émigrés de romans, c'est par l'écriture que le duc ressaisit le rapport à l'HISTOIRE, mais eux ont sur lui l'avantage de pouvoir - privilèges de la fiction que la nuit du 4 août n'a pas abolis refuser le cours d'une HISTOIRE qui les nie. Du moins en tant que classe,

(42) François-René de ChateAubriAnd, Mémoires d'outre-tombe, I, op. cit., p. 467. 
mais qui - et c'est aussi ce qui assure le contact avec le romanesque comme avec le romantisme -, nouveau statut de l'individu oblige, libère le moi et sa parole.

On reconnaît là une tension qui tient à un phénomène bien mis en évidence par Lukács, mais pour le roman ${ }^{43}$. Or ces analyses dépassent assurément le cadre générique dans la mesure où elles ne tiennent nullement aux catégories du récit, mais bien à celle de la personne, si bien que leur pertinence déborde la seule perspective narratologique, qu'on ne saurait comprendre en dehors de la référence au sujet et à la montée de l'individualisme, corrélatif de celui de la bourgeoisie. Les forces qui, chez Lukács, écartèlent son héros problématique sont celles-là mêmes qui divisent Gaston de Lévis et qui découvrent, par d'autres voies, la dialectique du moi et du monde en termes qui ne sont plus de simple extériorité, quand l'œuvre devient le creuset problématique de la belligérance entre individu et HISTOIRE : «Si j'étais destiné à vivre, je représenterais dans ma personne, représentée dans mes mémoires, les principes, les idées, les événements, les catastrophes, l'épopée de mon temps, d'autant plus que j'ai vu finir et commencer un monde, et que les caractères opposés de cette fin et de ce commencement se trouvent mêlés dans mes opinions ${ }^{44}$ avance le Chateaubriand des Mémoires.

Et Lévis, comme Chateaubriand, sera destiné à vivre. Si « Ce n'est pas vivre que d'exister dans un temps comme celui-ci » ${ }^{45}$, il connaîtra une seconde vie, expression de l'épopée de son temps, celle des Roland arcboutés sur leur pré carré. Appelé à la pairie dès la première Restauration, loin que «L'ambition » soit «finie » (4 août 1791, 360), il se porte à l'Académie française en 1816, où il succède à Roederer, exclu, dans le reniement de ce qu'il écrivait à Pauline en avril 1792: « je ne pense ni ne penserai à la gloire, c'est pour toi que j'écrirai, tu seras ma récompense » (389), mais dans la fidélité toujours à la lettre du romantisme, où les jeunes gens pleins de vertu et d'enthousiasme connaissent la triste fin des ralliés

(43) Georg LUKÁCS, Théorie du roman, Paris, Gallimard, « TEL », trad. franc. 1968 [1920].

(44) François-René de ChateAubriAnd, Mémoires d'outre-tombe, I, op. cit., p. 1046.

(45) C'est ce qu'écrit le 1er août 1791, de Paris, le duc de Lévis à sa jeune femme expatriée en Angleterre, p. 357. 
que Lucien Leuwen, écœuré de l'universelle curée de places et d'ambitions, flétrira de ce mot cruel : « Heureux les héros morts avant $1804 ! »^{46}$.

Laure LÉVÊQUE

BABEL (EA 2649)

Université de Toulon

Avenue de l'université

83957 La Garde

laure-leveque@wanadoo.fr

(46) STENDHAL, Lucien Leuwen, dans Romans et nouvelles I, Paris, Gallimard, « Bibliothèque de la Pléiade », 1952, p. 785. 